\title{
Effectiveness and safety of ShenXiong glucose injection for acute ischemic stroke: a systematic review and GRADE approach
}

\author{
Xue-ting Liu, Peng-wei Ren, Le Peng, De-ying Kang ${ }^{*}$, Tian-le Zhang, Shu Wen, Qi Hong and Wen-jie Yang
}

\begin{abstract}
Background: To appraise critically whether published trials of ShenXiong glucose injection for patients with acute ischemic stroke (AIS) are of sufficient quality, and in addition to rate the quality of evidence by using the GRADE approach (grading of recommendations, assessment, development, and evaluation, GRADE).

Methods: A literature search was performed in the Cochrane Library, MEDLINE, EMBASE, CBM, Chinese TCM (traditional Chinese medicine) Database, CNKI, VIP, WanFang Databases until January 2015. The limits were patients with AIS and randomized controlled trials (RCTs) or quasi-RCTs. Studies by which patients suffering intracerebral haemorrhage were excluded.

Results: Twelve studies fulfilled the inclusion criteria. We found significant benefits of ShenXiong glucose injection compared with conventional treatment in improving activities of daily living function at 4 weeks ( $\mathrm{MD}=34.12,95 \% \mathrm{Cl}$ : 29.07, 39.17), neurological function deficit at 2 weeks ( $M D=-5.39,95 \% \mathrm{Cl}:-6.90,-3.87)$, 4 weeks ( $\mathrm{MD}=-5.16$, $95 \% \mathrm{Cl}:-6.49,-3.83$ ), and clinical effects at 4 weeks ( $\mathrm{RR}=1.17,95 \% \mathrm{Cl}: 1.10,1.24)$. No trials reported the effects of ShenXiong glucose injection on the risk of early, deterioration, or quality of life. No adverse events were reported within the whole follow-up period.

Conclusions: The use of ShenXiong glucose injection may improve rehabilitation for patients with acute ischemic stroke, however, as the GRADE approach indicated low to moderate quality of available evidence as well as insufficient information about harm and patients preference, the recommendations were not provided for ShenXiong glucose injection taking as a therapeutic intervention to patients with acute ischemic stroke.
\end{abstract}

Keywords: ShenXiong glucose injection, Acute ischemic stroke (AIS), Systematic review (SR), GRADE

\section{Background}

Stroke occurs when the blood supply to the brain is interrupted or reduced, which may be caused by blocked arteries or leaking and bursting of blood vessels [1]. This deprives the brain of the supply of oxygen and nutrients, causing damage to the brain tissue [2]. Being one of the most severe neurological diseases, stroke often leads to death or gross physical impairment or disability [2], and the morbidity of stroke continues to rise with an annual increase of $8.1 \%$. Although the incidence of stroke is declining in many developed countries, the absolute number of stroke patients continues to enlarge because

\footnotetext{
* Correspondence: deyingkang@126.com

Department of Evidence-based Medicine and Clinical Epidemiology, West China Hospital, Sichuan University, Chengdu 610041, P. R. China
}

of the ageing population [3]. Of those, acute ischemic stroke as the dominant type of stroke, account for $85 \%$ of all strokes [4].

Currently, rapid assessment and early intervention for patients suffering an acute ischemic stroke is of top priority $[5,6]$. There is as yet no routine, effective, generally accepted, specific treatment for AIS, except for aspirin [7] and anti-thrombolysis with tissue plasminogen activator approved by FDA of US is administered in the setting of AIS within $4.5 \mathrm{~h}$ from symptom-onset [8-10]. Therefore, it is necessary to test other promising therapeutic approaches to acute ischemic stroke $[11,12]$. The lack of effective and widely applicable pharmacological treatments for acute ischemic stroke patients leads to a growing interest in Chinese traditional medicine [13, 14], 
for which extensive observational and anecdotal experience has accumulated over the past one thousand years $[14,15]$. Of those, Chinese herbal medicine is the most addressed in China, US and European Union [12, 13]. In China, a series of optional treatments for treating AIS are available. Traditional Chinese medicine have proven to be effective complementary intervention for AIS, of which Chinese herb products are still prevailing well and make a significant difference $[12,14,15]$. The overall treatment concept for Chinese traditional medicine is different from Western medicine, and herb medicines were always used to treat acute ischemic stroke. ShenXiong glucose injection is one of the most widely used Chinese herb medicine.

ShenXiong glucose injection (also named as DanshenChuanxiongqin injection) has properties that might be effective in acute ischemic stroke [16], it is on the national essential drug list of China as a new drug for cardio-cerebral vascular diseases. ShenXiong glucose injection is a compound preparation, made up of Salvia miltiorrhiza and Ligustrazine [16]. Of those, fifteen kinds of biologically active substances have been extracted from the dried roots of Salvia miltiorrhiza, a traditional medical herb known as Danshen, among which tanshinone, isotanshinone and hydroxy-tanshinone are the important ingredients [16, 17]. Various studies suggested that Danshen could decrease intracranial pressure by decreasing overall water content of the brain and cerebrospinal fluid volume, and by reducing blood volume due to vasoconstriction $[18,19]$. DanShen may also improve cerebral perfusion by decreasing viscosity. It is reported to increase coronary blood flow, suppress thromboxane formation, decrease cerebral oedema, infarct size and neurological deficit [19]. It is always used either alone or in combination with other herbal ingredients for patients with coronary heart diseases, hyperlipidemia, cerebrovascular diseases. In both China and other countries like US, such findings come either from biomedical studies [19] or from clinical trials [19, 20]. Ligustrazine, a bioactive ingredient extracted from Chuanxiong (Rhizoma Chuanxiong), was also proved to have beneficial effect on cerebrovascular diseases in pharmacological studies [21]. ChuanXiong could improve brain microcirculation by preventing thrombus formation and platelet aggregation and blood viscosity in order that it might have significant beneficial effects in the treatment of acute ischemic stroke [21]. As the compound of Salvia miltiorrhiza and ligustrazine, ShenXiong glucose injection has been widely accepted as a standard treatment for acute ischemic stroke in China for over 15 years [16]. Its mechanism is associated with exciting histamine receptors, improving neuron function and reducing serum total cholesterol-triglyceride levels, increasing serum high density lipoprotein levels, and enhancing tissue plasminogen activator activity. Moreover, ShenXiong glucose injection can prevent free radical injury from ischemic brain tissue by reducing lipid peroxide [16]

Currently there are a large number of studies of the clinical efficacy of ShenXiong glucose injection in acute ischemic stroke published in the global, especially in China. However, those clinical trials have not been reviewed systematically at present, whether the existing evidence is of sufficient scientifically rigorous and whether ShenXiong glucose injection can be recommended for routine use based on current evidence is still uncertain. To make specific recommendations for further research into the effectiveness and safety of ShenXiong glucose injection for patients with acute ischemic stroke, we carry out a systematic review and GRADE approach in twofold: (i) to systematically review all the randomized and quasirandomized controlled trials of ShenXiong glucose injection for acute ischemic stroke; (ii) in addition, to evaluate the quality of current evidence by the use of GRADE system, and to provide the best available evidence for clinical practice.

\section{Methods \\ Criteria for considering studies for this review and GRADE approach Type of studies}

We included all randomized controlled trials (RCTs), and quasi-randomized controlled trials (trials that used a nonrandom method of treatment allocation, e.g. hospital number, date of birth or day of the week), either published or unpublished.

\section{Types of participants}

Trials involving patients of any age or sex with apparent acute ischemic stroke (excluding intracerebral hemorrhage) were eligible. Stroke must be diagnosed in accordance with the diagnostic criteria of neuro-imaging verification of pathos-logical alterations in the brain, with computed tomography (CT) or Magnetic Resonance Imaging (MRI). Of these criteria, "Key Points for Diagnosing Cerebrovascular Diseases" and "Stroke diagnosis curative standard" modified in the 4th National Cerebrovascular Disease Seminar by the China Medical Society in 1995 [22] and "Guidelines of diagnosis and treatment of Chinese acute ischemic stroke" in 2010 [23], were adapted.

\section{Type of interventions}

Regardless of frequency, intensity or the duration of treatment, either ShenXiong glucose injection versus placebo or ShenXiong injection plus conventional treatments versus conventional treatments alone were included in this review and GRADE approach.

Any other routine treatments except ShenXiong glucose injection, such as antihypertensive, anti-platelet aggregation 
and other symptomatic treatments if necessary, were defined as conventional treatment, and all participants either in experimental group or in controlled group would receive same conventional treatments during whole trial period.

\section{Type of outcome measures}

The primary outcome is death or dependency at the end of scheduled follow-up. Dependency is defined as severely dependent on others in activity of daily living, based on the definition of the Barthel Index scores 60 or less, or an Oxford handicap grade 3 to 6.

Secondary outcomes were: (1) Changes of neurological deficit after ShenXiong glucose injection treatment and at the end of scheduled follow up. The measures could concentrate on specific impairment (for instance, Motricity Index, Motor Assessment Scale) or global neurological deficit (for instance, the National Institute of Health Stroke Scale or the Neurological Function Deficit Score). The clinical neurological impairment score of stroke patients is measured by the National Institute of Health Stroke Scale (NIHSS) or the Neurological Function Deficit Score (NFDS). The neurological function deficit score changes, such as NFDS reduction rate over $18 \%$ was taken as curative effect standard [24]; (2) Quality of life (QOL) at the end of follow up, which is measured by the Nottingham Health Profiles or Spiter Quality of Life Index; (3) Adverse events including bleeding, dizziness, vomiting, allergic reaction, or other adverse events caused by ShenXiong glucose injection. The number of patients developing at least one adverse event listed above was estimated.

\section{Search strategies \\ Electronic Search}

We searched the Cochrane Central Register of Controlled Trials (CENTRAL, Ovid, from 1991 to January 2015), MEDLINE (PubMed, from 1966 to January 2015), EMBASE (Ovid, from 1966 to January 2015), CBM (Chinese Biomedicine Database, from 1978 to January 2015), Chinese TCM Database (from 1949 to January 2015), CNKI (China National Knowledge Infrastructure, until January 2015), VIP (Chinese Scientific Journal Database from 1989 to January 2015), WanFang Database (from 1998 to January 2015). The key words comprise DanshenChuanxiongqin, Danshen-Chuanxiongqin, ShenXiong glucose injection, Dan Xiong and ShenXiong injection were used as English and corresponding Chinese search terms to identify studies from aforementioned databases. Reference lists of all included studies were also searched for publications that meet the inclusion criteria.

\section{Searching other resources}

In order to search all the related studies, we also handsearched the following relevant journals of TCM (the last issues were set at January 2015): World Journal of Integrated Traditional and Western Medicine, Chinese Journal of Integrative Medicine, Journal of Chinese Integrative Medicine, Journal of Traditional Chinese Medicine and Journal of Beijing University of Traditional Chinese Medicine.

\section{Data collection and analysis Selection of studies}

Clinical trials were identified by screening the titles and abstracts of targets by two independent reviewers $(\mathrm{XL}, \mathrm{LP})$, and any disagreements were resolved through discussion and consultation with a third reviewer (DK).

\section{Data extraction and management}

Two reviewers (XL, LP) independently extracted data from studies that met the inclusion criteria using a pre-designed form, and disagreements were resolved by discussing with the third reviewer (DK). We planned to retrieve the following data: methods (study design and execution, method of randomization), participant characteristics (number of patients, age, sex, inclusion criteria, method of diagnosis), interventions (description of interventions given to each treatment group including, with or without other combined treatment), outcomes, methods of analysis (intentionto-treatment analysis or per-protocol analysis, or both) and statistical methods used.

\section{Assessment of risk of bias in included studies}

Two reviewers (XL, LP) independently assessed the methodological quality of each included studies using the "risk of bias" assessment tool outlined in the Cochrane Handbook for Systematic Reviews of interventions (Version 5.1.0) [25]. Any disagreements were resolved by discussion with the third reviewer (DK).

\section{Statistical analysis}

The Cochrane Review Manager software, RevMan 5.1 [26], was used to calculate treatment effects across trials. For discontinuous data, we used relative risk (RR) with the corresponding $95 \%$ confidence interval (CI) as the measure of effect. For continuous data, the measure of the treatment difference for any outcome would be the mean difference (MD) when the pooled trials using the same rating scale or test, otherwise, the standardized mean difference (SMD) with $95 \%$ confidence interval (CI) would be used. A Chi-square based test of homogeneity was performed using Cochrane Q statistic [27] and $\mathrm{I}^{2}$. If $P$ value less than 0.10 or $\mathrm{I}^{2}$ value exceed $50 \%$ substantial, indicated heterogeneity among trial results 
significantly, we would carry out a subgroup analysis to explore the sources of heterogeneity, including clinical heterogeneity, such as the route of administration, time and dose interval of start of treatment, type of intervention, as well as the methodological heterogeneity, such as design type of the trials, the risk bias of happening and so on. If $\mathrm{I}^{2}$ was less than or equal to $50 \%$ we would use a fixed-effect meta-analysis, otherwise, if the underline true effect being assumed to follow up a normal distribution, we would use a random-effects model (in which case the confidence intervals will be broader than those of a fixed-effect model). Sensitivity analysis was carried out by using the leave-one-out approach to explore the influence of heterogeneity, to determine how robust the results of analysis were. Funnel plots and Egger analysis were used to assess the existence of publication bias, if possible.

Besides, in the process of interpreting results and coming to conclusions, we planned to use GRADE approach (grading of recommendation assessment, development, and evaluation, GRADE) to evaluate the quality of evidence. This approach is a systematic method for evaluating quality of evidence and formulating strength of recommendation if sufficient information is available [28]. Firstly, we subsequently classified the importance of each outcome. Three categories of outcome were specified based on their importance (median score of 1 to 9). Then the quality of evidence is divided into four categories: high, moderate, low, and very low. The GRADE approach defines clearly the methodological criteria by which evidence can be upgrade or downgrade and the justification for the strength of recommendation being formulated as "strong" or "weak". Critical and important outcomes were used for decision making and were included in the evidence profile and summary of Findings Tables [29].

\section{Results}

\section{Literature Search}

A flow diagram of study selection is shown in Fig. 1. After initially identifying 2949 articles, 12 studies that met the inclusion criteria were included for this review and GRADE approach (Table 1).

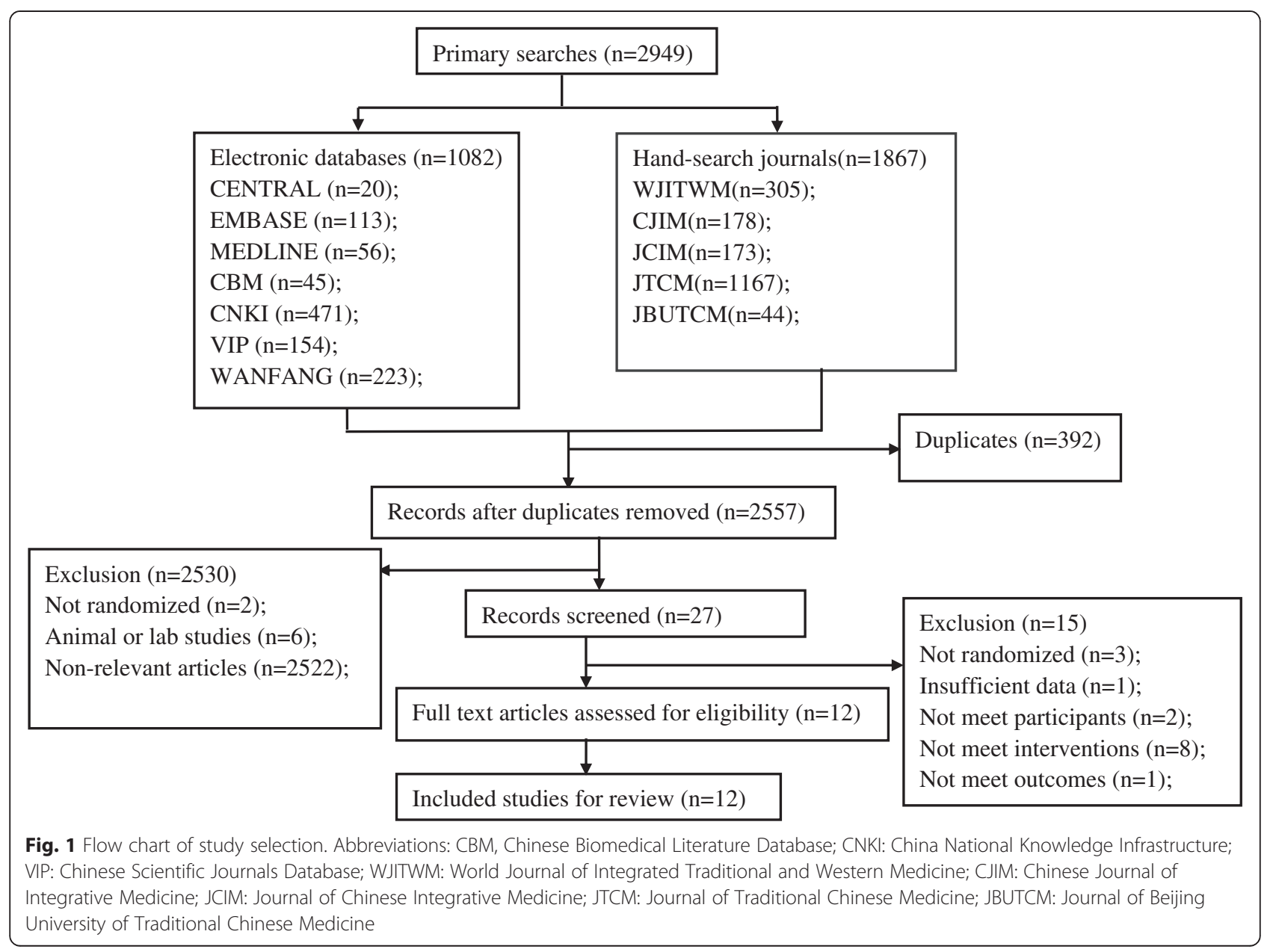


Table 1 Characteristics of included studies

\begin{tabular}{|c|c|c|c|c|c|c|c|c|c|c|c|}
\hline \multirow[t]{2}{*}{ First author } & \multicolumn{2}{|c|}{$\begin{array}{l}\text { Number of } \\
\text { patients (n) }\end{array}$} & \multicolumn{2}{|c|}{ Age (years, Mean \pm SD) } & \multirow[t]{2}{*}{$\begin{array}{l}\text { Diagnostic } \\
\text { criteria }\end{array}$} & \multirow[t]{2}{*}{$\begin{array}{l}\text { Onset } \\
\text { (days) }\end{array}$} & \multicolumn{2}{|c|}{$\begin{array}{l}\text { Baseline NFDS } \\
\text { (Mean } \pm \text { SD) }\end{array}$} & \multirow[t]{2}{*}{ Dose of Experiment } & \multirow[t]{2}{*}{ Conventional measure $(\mathrm{CM})$} & \multirow[t]{2}{*}{ Setting } \\
\hline & $\mathrm{E}$ & C & E & C & & & E & C & & & \\
\hline Wang 2014 [32] & 35 & 35 & $67.24 \pm 4.38$ & $68.15 \pm 5.0$ & $\mathrm{CT}, \mathrm{MRI}$ & $\leq 3 d$ & $18.15 \pm 8.14$ & $17.96 \pm 8.02$ & $\begin{array}{l}\text { Danshen Ligustrazine } \\
10 \mathrm{ml} / \mathrm{d}+250 \mathrm{ml} \text { glucose }+\mathrm{CM}\end{array}$ & $\begin{array}{l}\text { antihypertensive, anti platelet } \\
\text { aggregation }\end{array}$ & Inpatients \\
\hline Liu 2014 [30] & 71 & 71 & $53.1 \pm 1.7$ & $49.7 \pm 2.1$ & $\mathrm{CT}, \mathrm{MRI}$ & $\leq 3 d$ & $22.49 \pm 1.21$ & $23.18 \pm 1.74$ & $\begin{array}{l}\text { Danshen Ligustrazine } \\
13-15 \mathrm{ml} / \mathrm{d}+250 \mathrm{ml} \text { glucose }+\mathrm{CM}\end{array}$ & antihypertensive, reduce ICP & Inpatients \\
\hline Liu 2011 [39] & 34 & 33 & $41-79$ & $40-80$ & $\mathrm{CT}, \mathrm{MRI}$ & $\leq 3 d$ & $17.2 \pm 2.0$ & $16.7 \pm 3.2$ & $\begin{array}{l}\text { Danshen Ligustrazine } 10 \mathrm{ml} / \mathrm{d}+250 \mathrm{ml} \\
\text { glucose + CM }\end{array}$ & $\begin{array}{l}\text { anticoagulation, nutrition } \\
\text { brain cells }\end{array}$ & Inpatients \\
\hline Zhou 2013 [37] & 50 & 50 & $54.2 \pm 4.1$ & $54.2 \pm 4.1$ & NR & $\leq 3 d$ & NR & NR & $\begin{array}{l}\text { Danshen Ligustrazine } 10 \mathrm{ml} / \mathrm{d}+250 \mathrm{ml} \\
\text { glucose + CM }\end{array}$ & $\begin{array}{l}\text { Anti-platelet aggregation, } \\
\text { reduce ICP }\end{array}$ & Inpatients \\
\hline Huang 2012 [40] & 38 & 30 & $64.3 \pm 5.6$ & $66.3 \pm 5.3$ & $C T, M R I$ & NR & NR & NR & ShenXiong glucose $200 \mathrm{ml}$ plus CM & nutrition brain cells, reduce ICP & Inpatients \\
\hline Zhou 2011 [34] & 40 & 40 & $42-75$ & $42-75$ & $\mathrm{CT}, \mathrm{MRI}$ & NR & $22 \pm 10$ & $23 \pm 10$ & ShenXiong glucose $200 \mathrm{ml}$ plus CM & antihypertensive, reduce ICP & Inpatients \\
\hline Shan 2013 [31] & 30 & 30 & 65 & 64 & $C T, M R I$ & $\leq 3 d$ & $22.50 \pm 8.03$ & $21.65 \pm 6.2$ & ShenXiong glucose $200 \mathrm{ml}$ plus CM & $\begin{array}{l}\text { antihypertensive, anti platelet } \\
\text { aggregation }\end{array}$ & Inpatients \\
\hline Zhe 2011 [33] & 30 & 31 & $57.34 \pm 8.97$ & $58.26 \pm 7.48$ & $C T, M R I$ & $\leq 2 \mathrm{~d}$ & $24.63 \pm 9.28$ & $23.68 \pm 7.89$ & ShenXiong glucose $200 \mathrm{ml}$ plus CM & $\begin{array}{l}\text { nutrition brain cells, reduce ICP, } \\
\text { anti-platelet aggregation }\end{array}$ & Inpatients \\
\hline Ling 2013 [41] & 40 & 40 & $56.3 \pm 12.5$ & $58.3 \pm 11.8$ & $C T, M R I$ & $\leq 3 d$ & NR & NR & $\begin{array}{l}\text { ShenXiong } 10 \mathrm{ml} / \mathrm{d}+250 \mathrm{ml} \\
\text { glucose }+\mathrm{CM}\end{array}$ & $\begin{array}{l}\text { anti platelet aggregation, } \\
\text { nutrition brain cells }\end{array}$ & Inpatients \\
\hline Guan 2011 [42] & 46 & 40 & $63 \pm 3$ & $63 \pm 3$ & $\mathrm{CT}, \mathrm{MRI}$ & NR & NR & NR & ShenXiong glucose $200 \mathrm{ml}$ plus CM & reduce ICP, nutrition brain cells & Inpatients \\
\hline Nie 2013 [35] & 35 & 35 & $55-71$ & $54-70$ & $\mathrm{CT}, \mathrm{MRI}$ & NR & $25.63 \pm 9.08$ & $24.68 \pm 7.59$ & ShenXiong glucose $200 \mathrm{ml}$ plus CM & $\begin{array}{l}\text { anti platelet aggregation, } \\
\text { nutrition brain cells }\end{array}$ & Inpatients \\
\hline Lin 2014 [36] & 43 & 43 & $55.6 \pm 9.31$ & $52.8 \pm 8.72$ & $\mathrm{CT}, \mathrm{MRI}$ & $\leq 3 d$ & NR & NR & $\begin{array}{l}\text { ShenXiong } 10 \mathrm{ml} / \mathrm{d}+250 \mathrm{ml} \\
\text { glucose }+\mathrm{CM}\end{array}$ & $\begin{array}{l}\text { anti-hypertensive, anti-platelet } \\
\text { aggregation }\end{array}$ & Inpatients \\
\hline
\end{tabular}




\section{Study characteristics and quality assessment}

Characteristics of the 12 trials included in this review were summarized in Table 1, the number of participants that received ShenXiong glucose injection treatment ranged from 30 to 71 (total 494), and the number of participants in the control groups ranged from 30 to 71 (total 480). In 12 studies, ShenXiong glucose injection plus conventional therapy was administered in experimental group, the dose of Danshen-Chuanxiongqin ranges from $10 \mathrm{ml} / \mathrm{d}$ to $15 \mathrm{ml} / \mathrm{d}$, and the duration of treatment ranged from 2 to 4 weeks.

The results of the quality assessment of the included studies are shown in Table 2. Two studies [30,31] had low selection bias (allocating group by using random number table), two studies $[32,33]$ were quasi-randomized trials, other 8 trials reported "randomly allocating" participants, but not stated the method of randomization. Possible performance bias from inappropriate blinding of participants and personnel might be present in 12 studies.

\section{Effects of interventions \\ Death or dependency at the end of scheduled follow up period}

No deaths were reported within the period of treatment in 12 trials. This may mean that only mild strokes were included in the trials or that death occurred were not reported by trialists.

A measure of activities of daily living function was assessed in five [31-35] of the included trials. Barthel index scores was measured at 1 week in only one trial [32], 2 weeks in two studies [32, 34], and 4 weeks on treatment in four studies [31-33,35]. All of these trials reported sufficient information to allow for inclusion into a meta-analysis. Wang 2014 reported no significant difference on Barthel index score in patients randomized to ShenXiong glucose injection group as compared with control group, by approximately 7 days $(\mathrm{MD}=4.65, P=$ $0.14,95 \%$ CI: $-1.56,10.86)$; Two studies $[32,34]$ reported Barthel index score at 14 days, however, a pooled analysis was not performed since severe heterogeneity were detected between the trials $\left(P=0.02, \mathrm{I}^{2}=82 \%\right)$, which was possibly due to differences in outcome measurements used and types of treatment. The trial by Zhou 2011 reported that there was significant difference in the Barthel index scores at 14 days in the ShenXiong glucose injection group as compared with control group $(\mathrm{MD}=18.00,95$ \% CI: 11.43, 24.57). Trial by Wang 2014 reported no significant difference in the daily activities score at four weeks between the treatment groups ( $\mathrm{MD}=6.08,95 \% \mathrm{CI}:-1.19,13.35)$, but the confidence interval was very wide and included clinically significant effects in both directions (Fig. 2).

Four studies [31-33, 35] also assessed Barthel index scores at 28 days of treatment, however a pooled analysis was not performed since severe heterogeneity were detected among the trials $\left(\mathrm{I}^{2}=90 \%\right)$, which was possibly due to the differences in term of outcome measurements used and types of treatment. All of these trials reported higher Barthel scores at 28 days for the ShenXiong glucose injection group as compared with control group. We performed a subgroup analysis based on the treatment type (ShenXiong glucose injection [31, 33, 35] and Danshen ligustrazine injection [32]). There were significant difference in improvement of activities of daily living function

Table 2 Assessment of risk of bias in included studies

\begin{tabular}{|c|c|c|c|c|c|c|c|}
\hline Study & $\begin{array}{l}\text { Random Sequence } \\
\text { Generation }\end{array}$ & $\begin{array}{l}\text { Allocation } \\
\text { Concealment }\end{array}$ & $\begin{array}{l}\text { Blinding of Participants } \\
\text { and personnel }\end{array}$ & $\begin{array}{l}\text { Blinding of } \\
\text { Outcome assessment }\end{array}$ & $\begin{array}{l}\text { Incomplete } \\
\text { Outcome Data }\end{array}$ & $\begin{array}{l}\text { Selective } \\
\text { Reporting }\end{array}$ & $\begin{array}{l}\text { Other source } \\
\text { of bias }\end{array}$ \\
\hline Wang 2014 [32] & $\begin{array}{l}\text { sequence of } \\
\text { entering the group }\end{array}$ & Unclear & Unclear & Unclear & Yes & Yes & Unclear \\
\hline Liu 2014 [30] & $\begin{array}{l}\text { random number } \\
\text { table }\end{array}$ & Unclear & Unclear & Unclear & No & Unclear & Unclear \\
\hline Liu 2011 [39] & unclear & Unclear & Unclear & Unclear & Yes & No & Unclear \\
\hline Zhou 2013 [37] & unclear & Unclear & Unclear & Unclear & No & Yes & Unclear \\
\hline Huang 2012 [40] & unclear & Unclear & Unclear & Unclear & Yes & Unclear & Unclear \\
\hline Zhou 2011 [34] & unclear & Unclear & Unclear & Unclear & Yes & Yes & Unclear \\
\hline Shan 2013 [31] & $\begin{array}{l}\text { random number } \\
\text { table }\end{array}$ & Unclear & Unclear & Unclear & Yes & Yes & Unclear \\
\hline Zhe 2011 [33] & $\begin{array}{l}\text { sequence of } \\
\text { entering the group }\end{array}$ & Unclear & Unclear & Unclear & Yes & Yes & Unclear \\
\hline Ling 2013 [41] & unclear & Unclear & Unclear & Unclear & Yes & Unclear & Unclear \\
\hline Guan 2011 [42] & unclear & Unclear & Unclear & Unclear & No & Unclear & Unclear \\
\hline Nie 2013 [35] & unclear & Unclear & Unclear & Unclear & Yes & Unclear & Unclear \\
\hline Lin 2014 [36] & unclear & Unclear & Unclear & Unclear & Yes & Unclear & Unclear \\
\hline
\end{tabular}

Annotation: Yes = low risk of bias; No = high risk of bias; unclear = uncertain risk of bias 


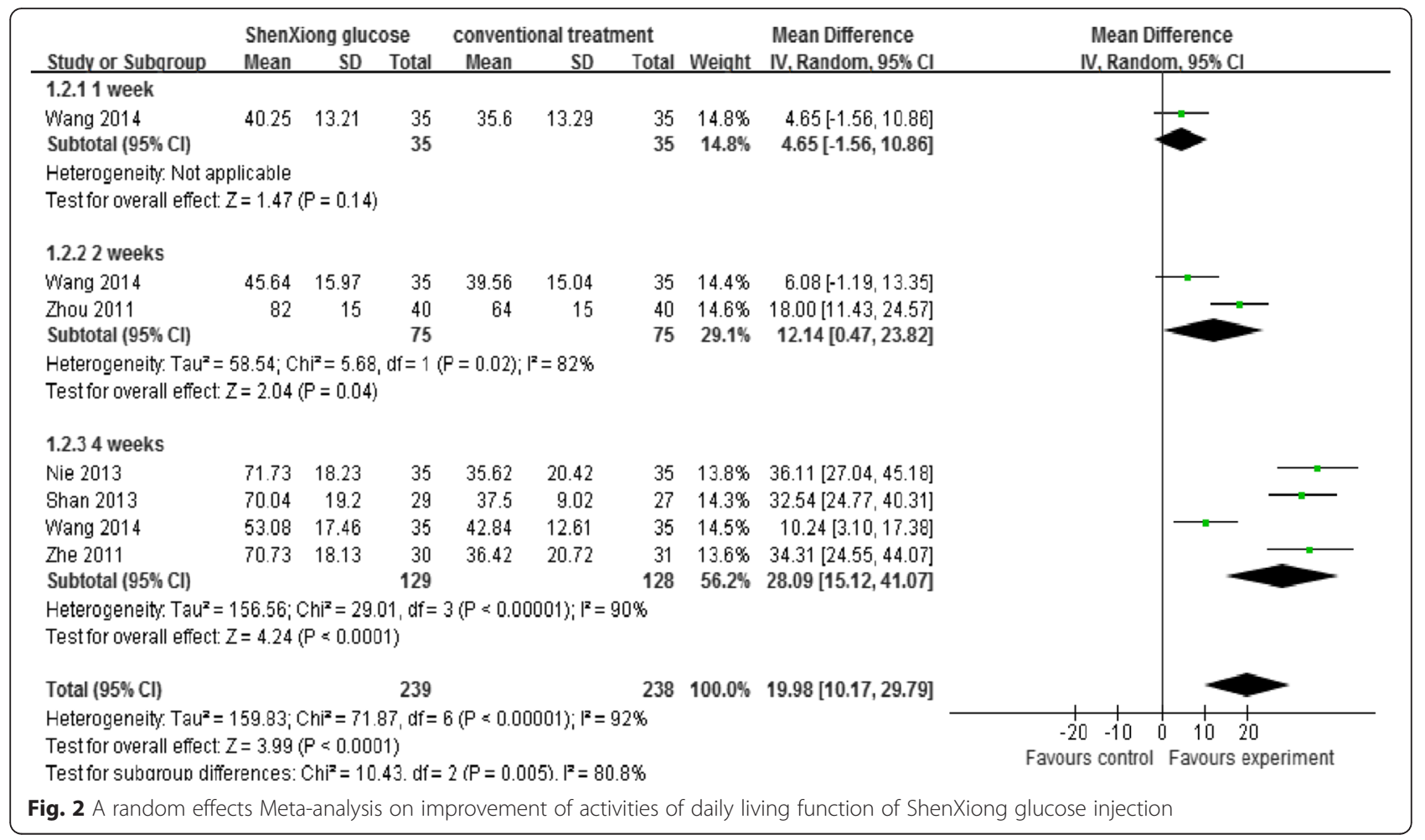

at 28 days in the ShenXiong glucose injection group compared with control group $(\mathrm{MD}=34.12$, $95 \% \mathrm{CI}$ : 29.07, 39.17); as well as in the Danshen ligustrazine injection group compared with control group (MD $=10.24$, $95 \% \mathrm{CI}$ : 3.10, 17.38) (Fig. 3).
Improvement of neurological function deficit after treatment and at the end of scheduled follow up Seven trials [30-36] with a total of 821 patients measured improvement of neurological function deficit after ShenXiong glucose injection treatment by using

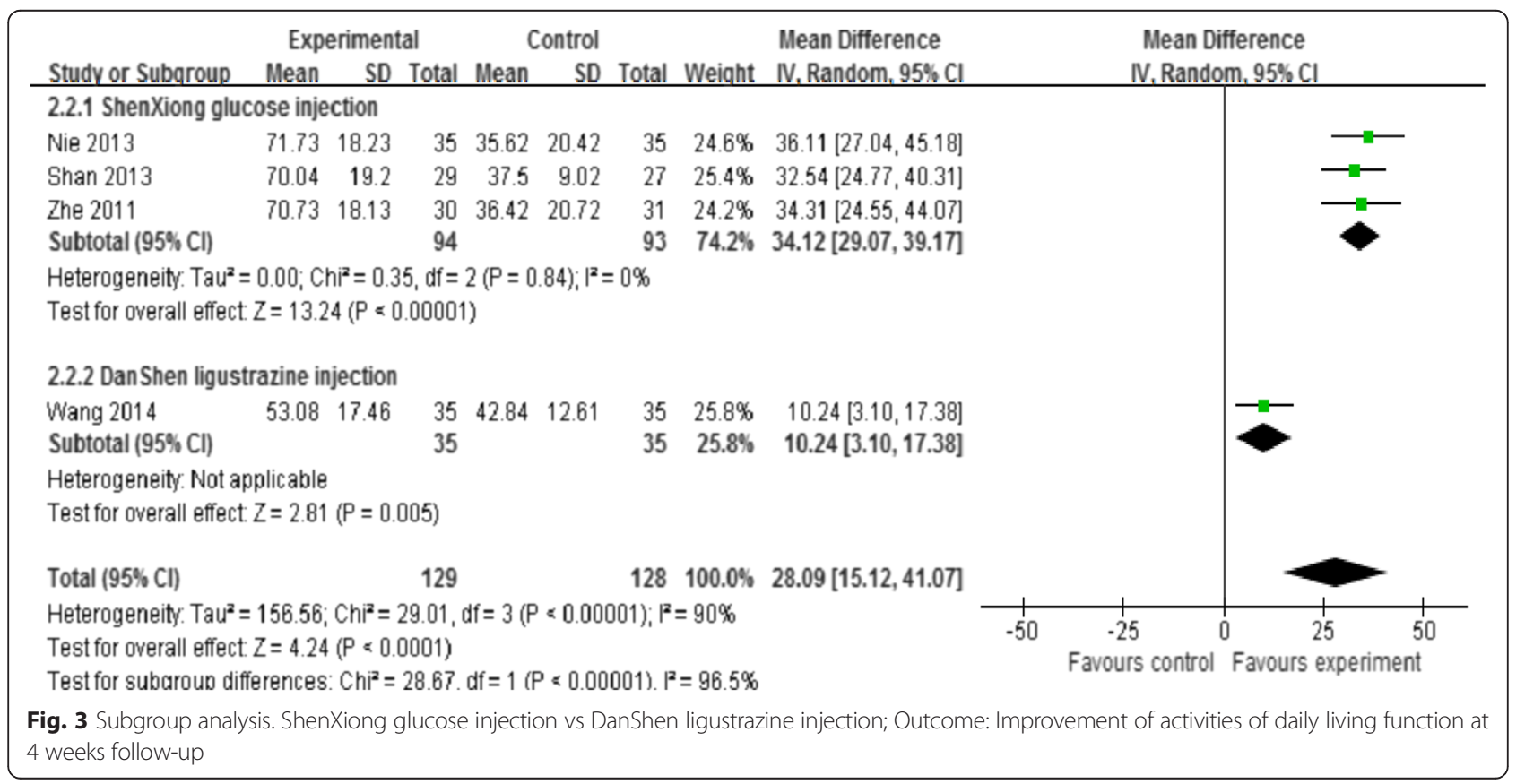


continuous approaches, such as changes of neurological deficit score. There was significant heterogeneity between studies [30,32] (Chi-squared $P=0.10, \mathrm{I}^{2}=63 \%$ ), which may be explained by the differences in outcome measurements used and types of treatment, therefore, the overall estimate of treatment effect should be interpreted with caution, although no significantly improvement of neurological impairment (MD for neurological improvement with ShenXiong glucose injection -2.37 , $95 \% \mathrm{CI}-5.44$ to 0.70 ) were observed at 7 days in the ShenXiong glucose injection group compared with control group (Fig. 4).

Four trials [30, 32, 36, 37] assessed improvement of neurological function deficit at 14 days on treatment, as there was significant heterogeneity among studies (Chi- squared $P=0.005, \mathrm{I}^{2}=77 \%$ ), which was possibly due to the differences in outcome measurements used and types of treatment. A subgroup analysis based on the treatment type (ShenXiong glucose injection [36] and Danshen ligustrazine injection [30, 32, 37]) indicated that there were significant difference in improvement of neurological function deficit at 14 days in the ShenXiong glucose injection compared with control group ( $\mathrm{MD}=-3.60,95 \% \mathrm{CI}:-4.88,-2.32$ ), as well as in the Danshen ligustrazine injection group compared with control group ( $\mathrm{MD}=-6.01,95 \% \mathrm{CI}:-6.51,-5.50)$ (Fig. 5).

Four studies [31-33, 35] also assessed the improvement of neurological function deficit at 28 days. A pooled analysis of these studies found a significant difference in the neurological function deficit scores at 28 days between the treatment groups ( $\mathrm{MD}=-5.16,95 \% \mathrm{CI}:-6.49,-3.83)$, low level of heterogeneity was detected among the studies $\left(P=0.31, \mathrm{I}^{2}=16 \%\right)$ (Fig. 4).

\section{Response rate}

The response rate taken as a measure of clinical effects was presented in eleven trials involving of 884 patients, there was no statistically significant heterogeneity in those studies $\left(P=0.81, \mathrm{I}^{2}=0 \%\right)$. Meta-analysis of 11 trials showed that ShenXiong glucose injection had significantly higher response rate than that of control group $(\mathrm{RR}=1.17,95$ \% CI: 1.10, 1.24) (Fig. 6).

\section{Safety evaluation}

Six trials clearly reported that there were no adverse events during the trials, while the left studies failed to report whether adverse events happening or not. The meta-analysis didn't perform due to numerical data for the outcomes of interest were unavailable.

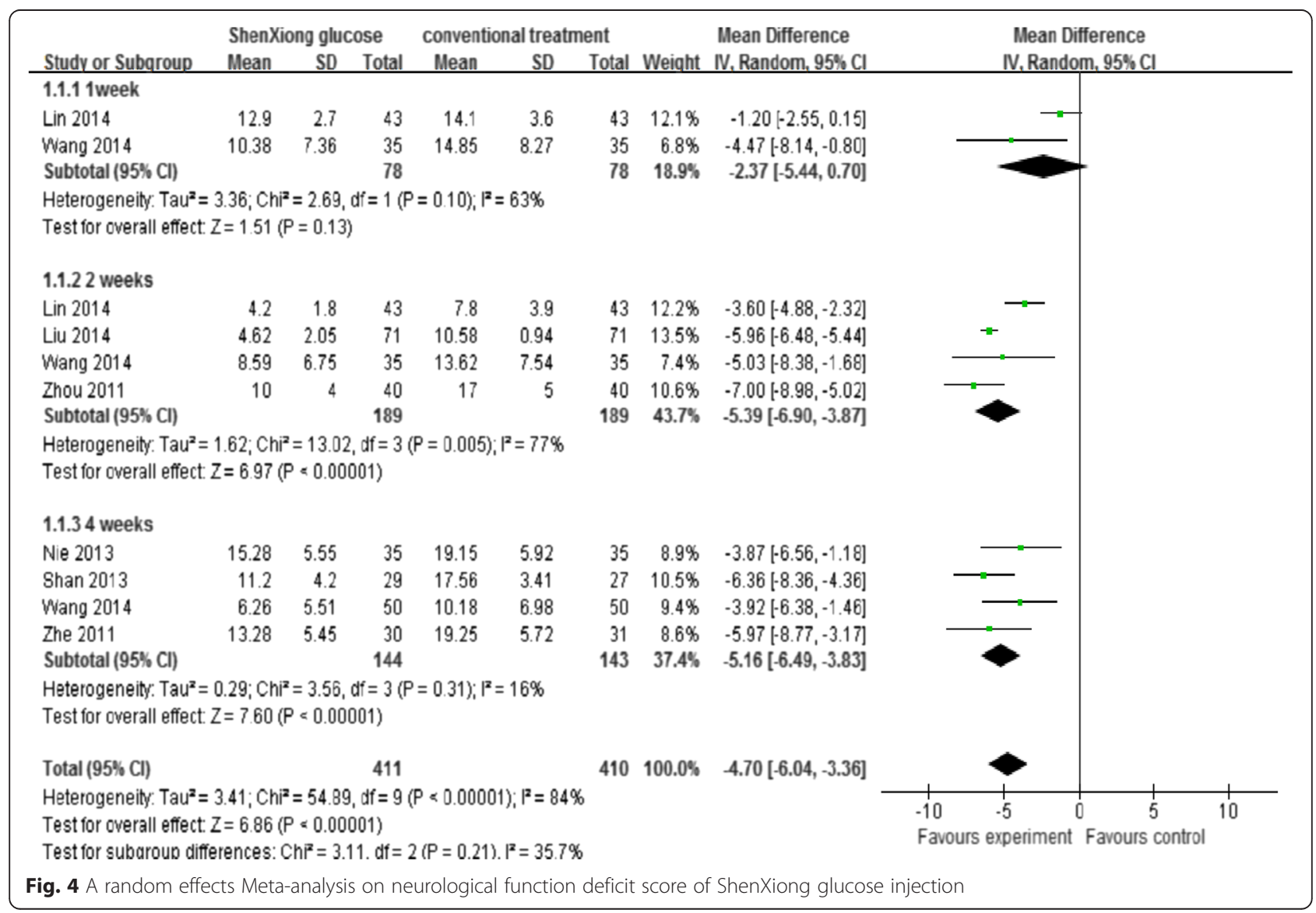




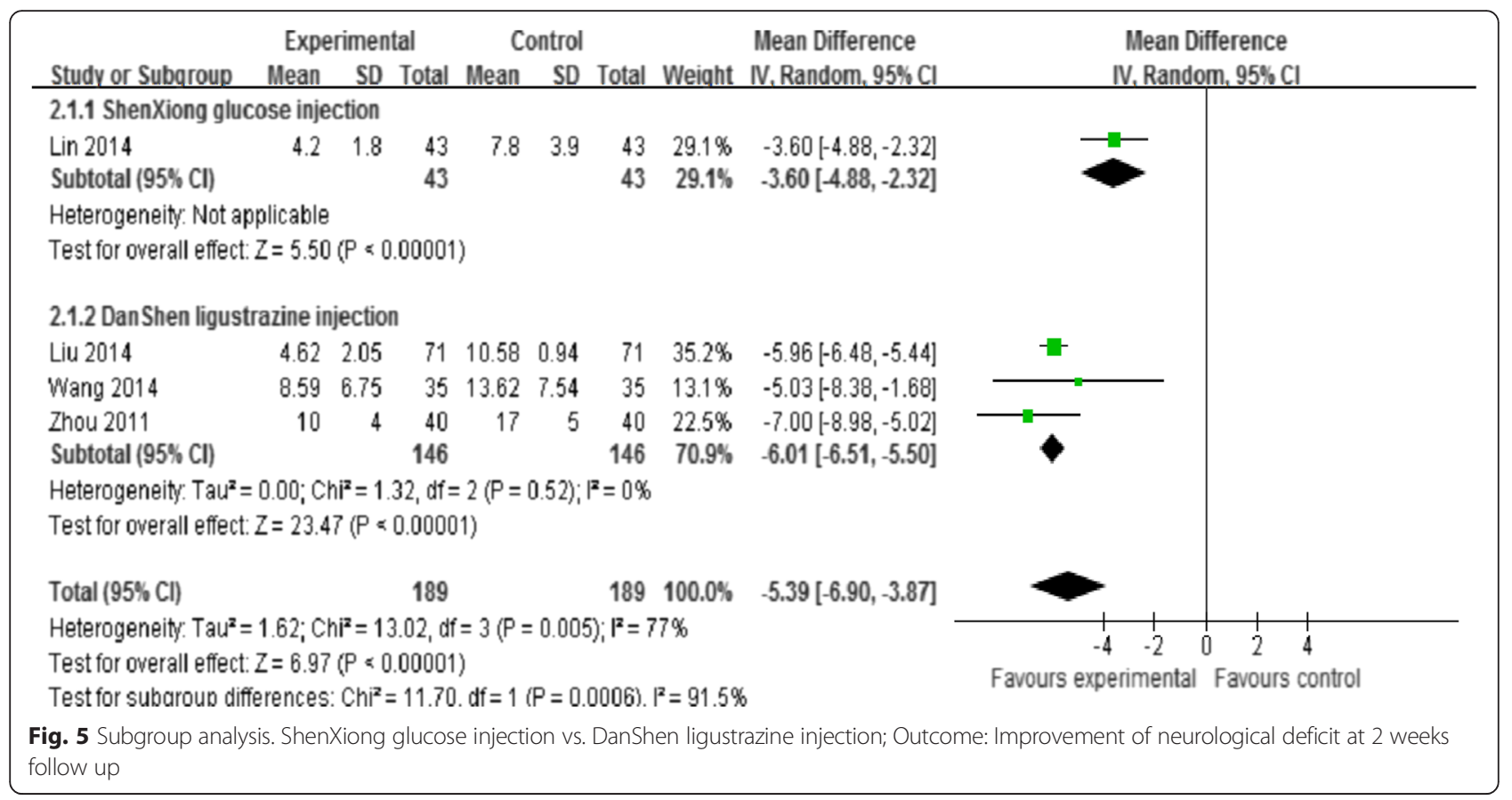

\section{Publication bias}

A funnel plot analysis on response rate of 11 trials was generated to explore the potential publication bias, and it present asymmetric trend, indicated that potential publication bias may exist in this review (Fig. 7).
The quality of evidence by using GRADE

In addition, we applied GRADE to grade the quality of available evidence, regarding to this GRADE approach, all 12 RCTs started out as high-quality evidence. Of those, seven trials [30-36] reported the improvement of

\begin{tabular}{|c|c|c|c|c|c|c|c|c|}
\hline \multirow[b]{2}{*}{ Study or Subqroup } & \multicolumn{2}{|c|}{ ShenXiong glucose } & \multicolumn{2}{|c|}{ conventional treatment } & \multirow[b]{2}{*}{ Weight } & \multirow{2}{*}{$\begin{array}{c}\text { Risk Ratio } \\
\text { M-H, Random, 95\% Cl }\end{array}$} & \multirow{2}{*}{\multicolumn{2}{|c|}{$\begin{array}{c}\text { Risk Ratio } \\
\mathrm{M}-\mathrm{H}, \text { Random, } 95 \% \mathrm{Cl}\end{array}$}} \\
\hline & Events & Total & Events & Total & & & & \\
\hline Guan 2011 & 43 & 46 & 30 & 40 & $9.3 \%$ & $1.25[1.03,1.51]$ & & - \\
\hline Huang 2012 & 35 & 38 & 25 & 30 & $10.3 \%$ & $1.11[0.92,1.33]$ & & + \\
\hline Ling 2013 & 39 & 40 & 33 & 40 & $15.4 \%$ & $1.18[1.02,1.37]$ & & 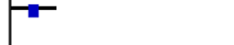 \\
\hline Liu 2011 & 30 & 34 & 24 & 33 & $6.0 \%$ & $1.21[0.95,1.55]$ & & \\
\hline Liu 2014 & 63 & 71 & 57 & 71 & $17.4 \%$ & $1.11[0.96,1.27]$ & & $t=$ \\
\hline Nie 2013 & 26 & 35 & 19 & 35 & $2.7 \%$ & $1.37[0.95,1.96]$ & & \\
\hline Shan 2013 & 26 & 29 & 22 & 27 & $7.4 \%$ & $1.10[0.88,1.37]$ & & \\
\hline Wang 2014 & 32 & 35 & 26 & 35 & $7.3 \%$ & $1.23[0.99,1.53]$ & & \\
\hline Zhe 2011 & 28 & 30 & 27 & 31 & $12.8 \%$ & $1.07[0.91,1.26]$ & & 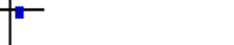 \\
\hline Zhou 2011 & 37 & 40 & 29 & 40 & $8.0 \%$ & $1.28[1.03,1.57]$ & & \\
\hline Zhou 2013 & 36 & 50 & 26 & 50 & $3.5 \%$ & $1.38[1.01,1.90]$ & & \\
\hline Total $(95 \% \mathrm{Cl})$ & & 448 & & 432 & $100.0 \%$ & $1.17[1.10,1.24]$ & & 1 \\
\hline Total events & 395 & & 318 & & & & & \\
\hline \multicolumn{7}{|c|}{ Test for overall effect: $Z=5.15(P<0.00001)$} & $\begin{array}{c}0.50 .7 \\
\text { Favours control }\end{array}$ & $\begin{array}{lll}1 & 1.5 & 2 \\
\text { Favours experiment }\end{array}$ \\
\hline
\end{tabular}




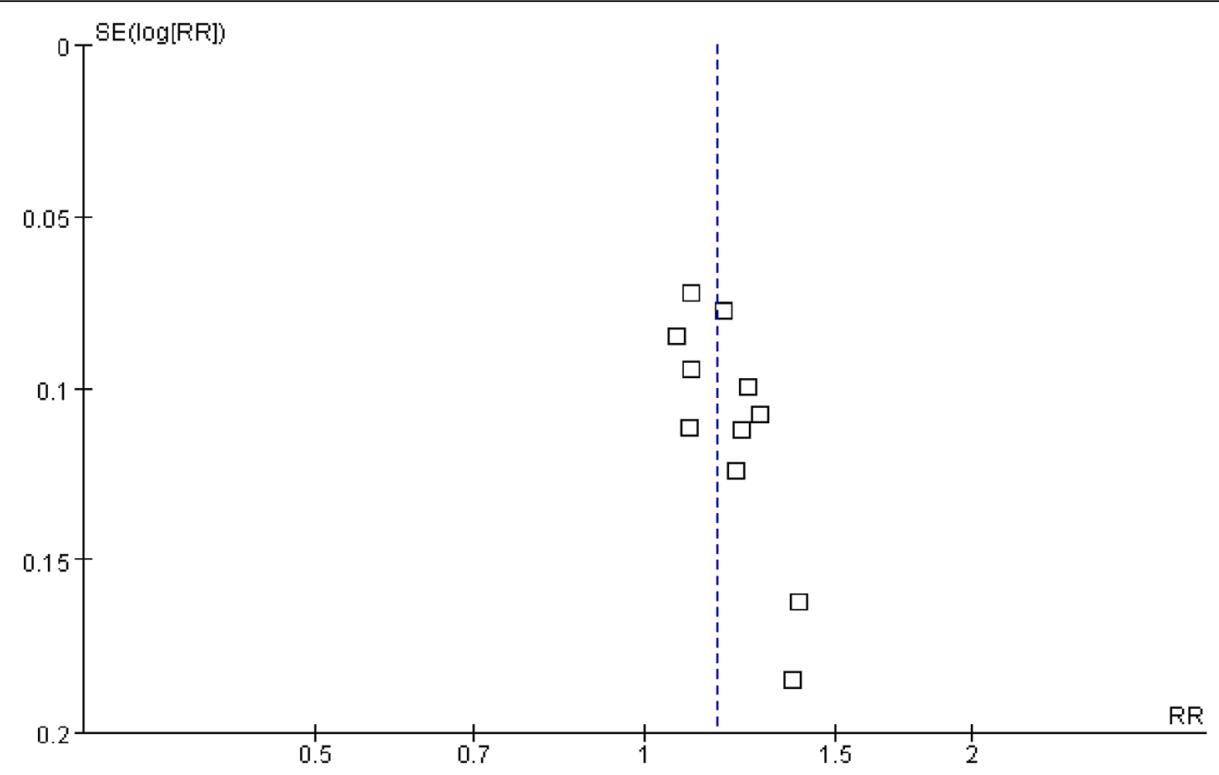

Fig. 7 Funnel plot analysis. Funnel plot analysis on response rate of the 11 trials comparing ShenXiong glucose injection with conventional treatment

neurological function deficit score (NFDS), the quality of the evidence was downgraded as moderate due to inconsistency and possible publication bias; Barthel index score was taken as critical outcome measure in five RCTs [31-35], the low quality was graded due to imprecision, inconsistency, and significant heterogeneity; eleven studies evaluated clinical effects by the use of response rate, and the evidence was set as moderate quality, publication bias and the lack of directness were the main considerations for downgrading (Table 3 and Additional file 1).

\section{Discussion}

\section{Summary of main findings}

This review was done in accordance with the methods of a Cochrane systematic review. We identified and included 12 trials involving a total of 974 participants. This systematic review has shown that ShenXiong glucose injection given as a treatment in the acute stage of ischemic stroke significantly effect on improving activities of daily living function at 7 days, 14 days and 28 days whilst on treatment, but a meta-analysis of this outcome using data from the five studies was not performed due to obvious heterogeneity among the study estimates. Significant improvements on activities of daily living function were observed from individual trials' results. We performed subgroup analysis based on the type of treatment (ShenXiong glucose injection and Danshen ligustrazine injection). No clear difference in Barthel index score at 28 days was present between ShenXiong glucose injection trial and Danshen ligustrazine injection trial. None of the included studies reported the pre-specified primary outcome of death during the follow-up period, it may be explained that authors attempted to emphasize short-term effect of ShenXiong glucose injection, or that there were no deaths during the short-term follow up period. Furthermore, pooled results showed ShenXiong glucose injection had significant effect on the improvement of neurological function deficit at 14-day and 28-day whilst on treatment. But this results should be interpreted with caution because of severe heterogeneity was seen among the review. We performed subgroup analysis based on the type of treatment (ShenXiong glucose injection and Danshen ligustrazine injection) to explore the reasons for large heterogeneity. It showed no evidence of difference in efficacy between ShenXiong glucose injection and Danshen ligustrazine injection. There is evidence that ShenXiong glucose injection has clinical effects, such as higher response rate, this result is consistent with the improvement of neurological function deficit. Lastly, only six trials reported that no adverse events were observed during the trials period, while the left studies failed to report whether adverse events happening or not. We cannot draw a conclusion on the safety of ShenXiong glucose injection. There is currently not enough evidence to determine whether the use of ShenXiong glucose injection in acute ischemic stroke would result in any harmful effects.

After applying GRADE approach for this systematic review, although rating down for the risk of bias was not required, Unexplained heterogeneity was found within included studies, while the available data did not permit an interpretation as to the reason for some of the inconsistencies we found, such inconsistency is also a basis for downgrading the level of evidence to support 
Table 3 ShenXiong glucose injection plus conventional treatment compared to conventional treatment for acute ischemic stroke

\begin{tabular}{|c|c|c|c|c|c|c|}
\hline \multirow[t]{3}{*}{ Outcomes } & \multicolumn{2}{|c|}{ Illustrative comparative risks ${ }^{\mathrm{a}}$ (95 \% Cl) } & \multirow{3}{*}{$\begin{array}{l}\text { Relative effect } \\
(95 \% \mathrm{Cl})\end{array}$} & \multirow{3}{*}{$\begin{array}{l}\text { No of Participants } \\
\text { (studies) }\end{array}$} & \multirow{3}{*}{$\begin{array}{l}\text { Quality of the } \\
\text { evidence (GRADE) }\end{array}$} & \multirow[t]{3}{*}{ Comments } \\
\hline & \multirow{2}{*}{$\begin{array}{l}\text { Assumed risk } \\
\text { Conventional } \\
\text { treatment }\end{array}$} & \multirow{2}{*}{$\begin{array}{l}\text { Corresponding risk } \\
\text { ShenXiong glucose plus } \\
\text { conventional treatment }\end{array}$} & & & & \\
\hline & & & & & & \\
\hline Death & none & none & none & none & none & CRITICAL \\
\hline $\begin{array}{l}\text { NFDS Follow-up: } \\
\text { 1-4 weeks }\end{array}$ & none & $\begin{array}{l}\text { The mean NFDS in the intervention } \\
\text { groups was }-4.70 \text { lower } \\
\text { (-6.04 to }-3.36 \text { lower) }\end{array}$ & - & $\begin{array}{l}821 \\
\text { (7 studies) }\end{array}$ & moderate & CRITICAL \\
\hline NFDS - 1 week & none & $\begin{array}{l}\text { The mean NFDS }-1 \text { week in the } \\
\text { intervention groups was }-2.37 \text { lower } \\
\text { ( }-5.44 \text { lower to } 0.7 \text { higher) }\end{array}$ & - & 156 (2 studies) & {$[1,2]$} & CRITICAL \\
\hline NFDS - 2 weeks & none & $\begin{array}{l}\text { The mean NFDS }-2 \text { weeks in the } \\
\text { intervention groups was }-5.39 \text { lower } \\
(-6.90 \text { to }-3.87 \text { lower })\end{array}$ & - & 378 (4 studies) & moderate [3-6] & CRITICAL \\
\hline NFDS - 4 weeks & none & $\begin{array}{l}\text { The mean NFDS }-4 \text { weeks in the } \\
\text { intervention groups was }-5.16 \text { lower } \\
\text { ( }-6.49 \text { to }-3.83 \text { lower })\end{array}$ & - & 287 (4 studies) & {$[7,8]$} & CRITICAL \\
\hline Barthel score & none & $\begin{array}{l}\text { The mean Barthel score in the } \\
\text { intervention groups was } 19.98 \text { higher } \\
\text { (10.17 to } 29.79 \text { higher) }\end{array}$ & - & 477 (5 studies) & low & CRITICAL \\
\hline $\begin{array}{l}\text { Barthel score - } \\
1 \text { week Follow-up: } \\
\text { mean } 1 \text { weeks }\end{array}$ & none & $\begin{array}{l}\text { The mean Barthel score }-1 \text { week in the } \\
\text { intervention groups was } 4.65 \text { higher } \\
(-1.56 \text { lower to } 10.86 \text { higher })\end{array}$ & - & 70 (1 study) & moderate [2] & CRITICAL \\
\hline $\begin{array}{l}\text { Barthel score - } \\
2 \text { weeks Follow-up: } \\
\text { mean } 2 \text { weeks }\end{array}$ & none & $\begin{array}{l}\text { The mean Barthel score }-2 \text { weeks in the } \\
\text { intervention groups was } 12.14 \text { higher } \\
\text { ( } 0.47 \text { to } 23.82 \text { higher) }\end{array}$ & - & 150 (2 studies) & {$[2,9]$} & CRITICAL \\
\hline $\begin{array}{l}\text { Barthel score - } \\
4 \text { weeks Follow-up: } \\
\text { mean } 4 \text { weeks }\end{array}$ & none & $\begin{array}{l}\text { The mean Barthel score - } 4 \text { weeks in the } \\
\text { intervention groups was } 28.09 \text { higher } \\
\text { ( } 15.12 \text { to } 41.07 \text { higher) }\end{array}$ & - & $\begin{array}{l}257 \\
\text { (4 studies) }\end{array}$ & {$[10,11]^{\text {low }}$} & CRITICAL \\
\hline \multirow{4}{*}{$\begin{array}{l}\text { Response rate } \\
\text { Follow-up: mean } \\
4 \text { weeks }\end{array}$} & \multicolumn{2}{|c|}{ Study population } & \multirow{4}{*}{$\begin{array}{l}\text { RR } 1.17 \\
\text { (1.1 to } 1.24)\end{array}$} & \multirow{4}{*}{$\begin{array}{l}880 \\
\text { (11 studies) }\end{array}$} & \multirow{4}{*}{ moderate [10] } & \multirow[t]{4}{*}{ IMPORTANT } \\
\hline & 736 per 1000 & 861 per 1000 (810 to 913) & & & & \\
\hline & Moderate & & & & & \\
\hline & 750 per 1000 & 878 per 1000 (825 to 930$)$ & & & & \\
\hline
\end{tabular}

Patient or population: patients with acute ischemic stroke Settings: inpatients, Intervention: ShenXiong glucose injection plus conventional treatment, Comparison: conventional treatment

${ }^{a}$ The basis for the assumed risk (e.g. the median control group risk across studies) is provided in footnotes. The corresponding risk (and its $95 \%$ confidence interval) is based on the assumed risk in the comparison group and the relative effect of the intervention (and its $95 \% \mathrm{Cl}$ )

Cl Confidence interval

RR Risk ratio

GRADE Working Group grades of evidence

$(\oplus \oplus \oplus \oplus)$ High quality: Further research is very unlikely to change our confidence in the estimate of effect

$(\oplus \oplus \oplus \ominus)$ Moderate quality: Further research is likely to have an important impact on our confidence in the estimate of effect and may change the estimate

$(\oplus \oplus \ominus \ominus)$ Low quality: Further research is very likely to have an important impact on our confidence in the estimate of effect and is likely to change the estimate $(\oplus \Theta \Theta \Theta)$ Very low quality: We are very uncertain about the estimate

[1] Heterogeneity test: $P=0.10, I^{2}=63 \%$. Obvious inconsistency exists in only two studies

[2] Imprecision (large width of $\mathrm{Cl}$ ). Sample sizes and number of events less than the number of patients generated by a conventional sample size calculation for a single adequately powered trial

[3] As the random sequence generation was not clearly described in only two of the trials, the overall risk of bias is not serious

[4] Heterogeneity test: $P=0.005 ; I^{2}=77 \%$. The available data do not permit an interpretation as to the reason for some of the inconsistencies that were found. Such inconsistency is also a basis for downgrading the level of evidence in support of ShenXiong glucose injection for AIS

[5] Although the sample sizes and number of events $(n=292)$ less than the number of patients generated by a conventional sample size $(n=300)$ calculation for a single adequately powered trial, the change of our confidence for this outcome not serious, thus not downgrade

[6] The funnel plot present asymmetric trend

[7] Although the sample sizes and number of events $(n=287)$ less than the number of patients generated by a conventional sample size ( $n=300)$ calculation for a single adequately powered trial, the overall imprecision for this outcome is not serious, thus not to downgrade the quality of evidence for imprecision [8] Heterogeneity: $P=0.02 ; I^{2}=82 \%$. The available data do not permit an interpretation as to the reason for some of the inconsistencies that were found. Such inconsistency is also a basis for downgrading the level of evidence

[9] Heterogeneity: $P<0.00001 ; I^{2}=90 \%$. The available data do not permit an interpretation as to the reason for some of the inconsistencies that were found. Such inconsistency is also a basis for downgrading the level of evidence

[10] The funnel plot present asymmetric trend on clinical efficacy, and the risk of publication bias may exist in somewhat

[11] Heterogeneity: $(P=0.39) ; 1^{2}=0 \%$, mild inconsistency maybe exists in 4 studies 
ShenXiong injection for AIS patients. In addition, regarding the two important outcomes, the total number of events or patients does not exceed the OIS (Optimal Information Size), therefore, rating down for imprecision was warranted [38]. By the way, the quality of evidence was not reduced in concerning indirectness. And finally, despite the fact that none of the included studies were found by pharmaceutical industry, the funnel plot present asymmetric trend on clinical efficacy, and the risk of publication bias may exist in somewhat.

Therefore, our GRADE approach leads to the decision that ShenXiong glucose injection for a individual patient with acute ischemic stroke should be approached cautiously, patients'values and preferences should be considered and allow them to participate in the medical decision making process.

\section{The limitations of the twelve trials assessed to be of inferior methodological quality were as follows}

1. Only two trials using a random method to divide the groups, the remaining 10 trials reported 'randomly allocating' participants but the method of randomisation was not described. 2. None of the included trials reported allocation concealment, and whether a blinding method used or not within twelve trials is unclear, increasing the risk of selection or performance bias happening. 3 . In addition, all included studies were conducted in China and were published in Chinese journals. A funnel plot analysis also indicated asymmetry trend for the outcome of response rate, publication bias maybe exist, although we attempt to perform comprehensive search as well as supplementary search for both published and unpublished literature. 4. Another limitation of the review relate to the over-proportion of small trials to be included, may cause imprecision and inconsistency of effect size among those trials. Although the estimate of effect on the outcomes is significant statistically, the observed effects could also simply be due to bias rather than to a biological effect of ShenXiong glucose injection.

In addition, the usefulness of evidence was limited due to the following reasons,. Firstly, the follow-up time was insufficient to evaluate the long term effects of ShenXiong glucose injection, in the 12 trials, follow-up time ranged from 7 days ( 1 week) to 28 days (4 week) after the start of treatment. Secondly, the reporting on adverse effect was poor in most of included trials, it is highly implausible that no adverse event happen among 494 patients, the safety of ShenXiong injection is still inconclusive. And additional limitations in this review include the diversity of outcome measure used by the included studies, and the limited duration of follow up (generally no more than 2 months).

In summary, the overall quality of evidence was judged to be low to moderate due to important limitations exist in study design, inconsistency, lack of directness, imprecision and possible publication bias. The result of published intervention trials are therefore of insufficient quality to enable evidence-based recommendations to be developed for clinical practice in acute ischemic stroke. This review systematically describes current evidence from latitudinal and longitudinal studies on ShenXiong glucose injection and identifies methodological aspects to be improved in further research. This methodological improvement is pivotal for the assessment of the body of evidence on ShenXiong glucose injection treatment. High-quality evidence from methodologically via double-blinded randomized controlled trials are required to provide further support for administration of ShenXiong glucose injection for patients with acute ischemic stroke.

\section{Conclusion}

\section{Implications for practice}

The results of this review provided evidence to support that the use of ShenXiong glucose injection may improve rehabilitation for patients with acute ischemic stroke, however, as the GRADE approach indicated low to moderate quality of available evidence as well as insufficient information about harm and patients preference, the recommendations for ShenXiong glucose injection for treating patients with acute ischemic stroke were not provided in this context.

\section{Implications for research}

Further RCTs on ShenXiong glucose injection are needed and should be reported according to the CONSORT (Consolidated Standards for Reporting Trials, CONSORT) statement. High quality on the reporting of trials is strongly required.

\section{Additional file}

Additional file 1: The PRISMA checklist. (DOC $62 \mathrm{~kb}$ )

\section{Competing interests \\ This work was supported by funds from State Administration of Traditional Chinese Medicine.}

\begin{abstract}
Authors' contribution
$\mathrm{DK}, \mathrm{PR}$ and $\mathrm{QH}$ conceived and designed the study, $\mathrm{XL}$ and $\mathrm{LP}$ developed search strategy, searched data, trial selected, data extraction, as well as contacting authors for additional data. XL, LT, WY and LP performed data analyses, presented results, and revised the manuscript. XL and SW interpreted results and draw conclusions. All authors declared that there are no conflicts of interest. All authors read and approved the final manuscript.
\end{abstract}

\section{Acknowledgements}

None.

Received: 1 July 2015 Accepted: 4 February 2016

Published online: 19 February 2016 


\section{References}

1. El Tecle NE, El Ahmadieh TY. Stroke: a global killer on the rise. World Neurosurg News. 2013;80:2-12.

2. Arun Paul A. Brain and vascular imaging of acute stroke. World Neurosurg. 2011;76:S3-8.

3. WHO2007a: World Health Organization. Global burden of stroke. http//www.who. int/cardiovascular diseases/en/cvd. Accessed July 2014.

4. Ministry of Health, PRC: Chinese Health Statistical Digest 1999:16:69-70.

5. Kan P, Dumont TM, Elad I. Acute stroke therapy: are we ready for primetime clinical trials? World Neurosurg. 2012;77:594-600

6. Fisher M, Schabitz UR. An overview of acute stroke therapy. Arch Intern Med. 2000;160:3196-206.

7. Wardlaw JM, del Zoppo G, Yamaguchi T: Thrombolysis for acute ischaemic stroke. Cochrane Database Syst Rev. 2009;10:1002.

8. Natarajan SK, Karmon Y. Prospective acute ischemic stroke outcomes after endovascular therapy: a real-world experience. World Neurosurg. 2010;4:455-64.

9. Taussky P, Tawk RG. Medical therapy for ischemic stroke: review of intravenous and intra-arterial treatment options. World Neurosurg. 2011;76:S9-S15.

10. The SPS3 Investigators. Effects of clopidogrel added to aspirin in patients with recent lacunar stroke. N Engl J Med. 2012;367:817-25.

11. Lawes CM, Bennett DA, Feigin VL. Blood pressure and stroke: an overview of published reviews. Stroke. 2004;35:1024-33.

12. Fetrow CW, Avita JR. Understanding and using herbal medicine. The Complete Guide to Herbal Medicine 2000;6:1-3.

13. Lu WI, Lu DP. Impact of Chinese herbal medicine on American society and health care system: perspective and concern. Evid Based Complement Alternat Med. 2014;10:1-6.

14. Feigin VL. Herbal medicine in stroke, does it have a future? Stroke. 2007; 38:1734-6.

15. Cheng TO. Danshen: A popular Chinese cardiac herbal drug. J Am Coll Cardiol. 2006:47:1487-501.

16. Min $\mathrm{H}$, Wang Q. The research progress of Salvia miltiorrhiza ligustrazine hydrochloride and glucose injection in clinical. Chin J Cerebrovasc Dis. 2013;7:41-3.

17. Dong J, Xu L. Beneficial effects of acetyl salvianolic acid A on focal cerebral ischemic rats subjected to middle cerebral artery thrombosis. Acta Pharmaceutica Sinica B. 1996;31:6-9.

18. Min L, Dang L, Ma W. Clinical study on effect and therapeutic mechanism of composite Salvia injection on acute cerebral infarction. Chin J Integr Tradit Western Med. 2002;22:353-5.

19. Cheng TO. Cardiovascular effects of DanShen. Int J Cardiol. 2007;121:9-22.

20. Ge L, Zhao W, Zhou Z. Danshen extracts decrease blood $C$ reactive protein and prevent ischemic stroke recurrence: a controlled pilot study. Phytother Res. 2009:23:1721-5.

21. Ni X, Liu S, Guo X. Medium- and long-term efficacy of ligustrazine plus conventional medication on ischemic stroke: a systematic review and meta-analysis. J Tradit Chin Med. 2013;33:715-20.

22. Neurology Branch of Chinese Medicine Association. Key points for diagnosing cerebrovascular diseases. Chin J Neurol. 1996;29:379-80.

23. Liu M, Zhang M, Li R. Guidelines of diagnosis and treatment of Chinese acute ischemic stroke in 2010. Chinese General Practice. 2011;12:4013-7.

24. The fourth national cerebrovascular disease conference. Cerebral apoplexy patients clinical nerve function defect score standard. The neurologist magazine. 1996:29:381-3.

25. Julian PT Higgins, Sally Green (Eds): Cochrane Handbook for Systematic Reviews of Interventions Version 5.1.0. The Cochrane Collaboration 2011, http://www.cochrane-handbook.org

26. The Nordic Cochrane Centre, The Cochrane Collaboration: Review Manager software (RevMan). Version 5.1. Copenhagen: the Nordic Cochrane Centre. The Cochrane Collaboration 2011.

27. Higgins JPT, Thompson SG. Quantifying heterogeneity in a meta-analysis. Stat Med. 2002;21:1539-58

28. Guyatt $\mathrm{GH}$, Oxman AD, Vist GE. GRADE: an emerging consensus on rating quality of evidence and strength of recommendations. BMJ. 2008;336:924-6.

29. Guyatt G, Oxman AD, Akl EA. GRADE guidelines: Introduction- GRADE evidence profiles and summary of findings tables. J Clin Epidemiol. 2011;64:395-400

30. Liu W, Xiao H, Zhou X. Clinical study of salviae miltiorrhizae and ligustrazine hydrochloride injection aided identification and treatment of ischemic stroke. Chin J Biochem Pharm. 2014;34:110-1.
31. Shan J, Li H. Clinical observation of Shenxiong glucose injection in treatment of acute ischemic stroke. Chin Manipulation Rehabil Med. 2013;3:23.

32. Wang A, Zhang Q. Salvia miltiorrhiza and ligustrazine injection on NFDS score and Barthel index of elderly patients with acute cerebral infarction. LIAONING J Tradit Chin Med. 2014;41:921-2.

33. Zhe $Y$, Nie $X$. Shenxiong glucose injection in treatment of acute ischemic stroke for 30 patients. China Pharm. 2011;20:78-9.

34. Zhou S. Study on Shenxiong glucose injection in treatment of cerebral infarction. Mod Prev Med. 2011:38:2656-9.

35. Nie $X$, Zhang $Y$. Shenxiong glucose injection in treatment of 40 patients with acute cerebral infarction. Nei Mongol J Tradit Chin Med. 2013:34:45-6.

36. Lin X, Wu H. Ai Ming: The effectiveness of Shao Danshen ChuanXiong Qin injection for the treatment of acute ischemic stroke. Chin J Health Lab Tec. 2014;24:3079-81.

37. Jingxia Z, Qingjie S, Chaosheng Z, Hairong W. Effects of Danshen Chuanxiongqin injection combined with conventional treatment in patients with cerebral apoplexy and blood rheology. Chin Med Guide. 2013;15:674-5.

38. Guyatt GH, Oxman AD, Kunz R, Brozek J, Alonso-Colello P, Rind D, et al. GRADE guideline 6. Rating the quality of evidence: imprecision. J Clin Epidemiol. 2011;64:1283-93.

39. Liu C. The effectiveness of Danshen-Chuanxiongqin injection for the treatment of acute ischemic stroke. Med Innov China. 2011:30:88-9.

40. Huang P, Shao W, Pan X. Clinical observation of Shenxiong glucose injection in treatment of acute cerebral infarction. Hubei J TCM. 2012:34:3-4.

41. Ling J, Liu Q, Wu J. The influence of Danshen ligustrazine injection for the patients with acute ischemic stroke. Res Integr Tradit Chin West Med. 2013;5:60-2.

42. Guan $\mathrm{L}$. The clinical observation of 86 patients with acute ischemic stroke with Shenxiong glucose injection. Chin J Mod Drug Appl. 2011;5:149-50.

\section{Submit your next manuscript to BioMed Central and we will help you at every step:}

- We accept pre-submission inquiries

- Our selector tool helps you to find the most relevant journal

- We provide round the clock customer support

- Convenient online submission

- Thorough peer review

- Inclusion in PubMed and all major indexing services

- Maximum visibility for your research

Submit your manuscript at www.biomedcentral.com/submit 